\title{
Model reduction of dissipative systems by balanced truncation
}

\author{
H. B. $\operatorname{Minh}^{*}$ H. L. Trentelman ${ }^{* *}$ P. Rapisarda*** \\ * Mathematics Institute, University of Groningen, P.O. Box 800, 9700 \\ AV Groningen, The Netherlands, h.b.minh@math.rug.nl \\ ** Mathematics Institute, University of Groningen, P.O. Box 800, \\ 9700 AV Groningen, The Netherlands, Phone:+31-50-3633999, \\ Fax:+31-50-3633800 h.l.trentelman@math.rug.nl \\ *** School of Electronics and Computer Science, University of \\ Southampton, SO17 1BJ, United Kingdom, pr3৫ecs.soton.ac.uk
}

\begin{abstract}
Given a dissipative system behavior with general supply rate, say a $\Sigma$-dissipative system, we propose a balanced truncation method to obtain a reduced-order system behavior which is again $\Sigma$-dissipative. This methods applies to driving variable representations of systems. A one-step error bound formula is discussed.
\end{abstract}

Keywords: Model reduction, strictly dissipative systems, driving-variable representation, balanced truncation.

\section{INTRODUCTION}

Model reduction by balanced truncation is well-known technoque. It is one of the best algorithms in the sense that it has a nice physical interpretation and that it preserves stability. Furthermore, error bounds exist. The method is well-studied for various systems, ranging from classical input/state/output systems to positive real, and bounded real systems (see Antoulas (2004), Chen et al. (1995), Opdenacker et al. (1988)). However in the behavioral context, there are still very few papers investigating this subject, see Willems et al. (2002); Rapisarda et al. (2003); Weiland (1991).

This paper aims to investigate this topic for the class of dissipative system behaviors. We show that dissipativity is preserved by reduction by balanced truncation; moreover, we provide an error bound. We work with driving variable representations. The main reason for this choice is that this class is still more general than the class of input/state/output representations, but no a priori inputoutput partition needs to be made. Furthermore, driving variable representations are state representations, having the advantage that truncation of the states can be performed.

Notation and background material. Throughout the paper we will denote by $\mathbb{R}(i \mathbb{R}, \mathbb{C})$ the space of real (pure imaginary, complex) number. We denote by $\mathfrak{C}^{\infty}\left(\mathbb{R}, \mathbb{R}^{\mathrm{w}}\right)$ the set of infinitely often differentiable functions from $\mathbb{R}$ to $\mathbb{R}^{\mathrm{w}}$, with $\mathfrak{D}\left(\mathbb{R}, \mathbb{R}^{\mathrm{w}}\right)$ the subspace of $\mathfrak{C}^{\infty}\left(\mathbb{R}, \mathbb{R}^{\mathrm{w}}\right)$ consisting of all compactly supported functions, with $\mathfrak{L}_{2}^{\text {loc }}\left(\mathbb{R}, \mathbb{R}^{\mathrm{w}}\right)$ the set of all Lebesgue measurable functions $w$ from $\mathbb{R}$ to $\mathbb{R}^{\mathrm{w}}$ for which the integral $\int_{\Omega}\|w\|^{2} d t$ is finite for all compact sets $\Omega \subset \mathbb{R}$.

We introduce now the definition of dissipativity of the system.
Definition 1. Let $\mathfrak{B} \in \mathfrak{L}_{\text {contr }}^{\mathrm{w}}$ and $\Sigma=\Sigma^{\top} \in \mathbb{R}^{\mathrm{w} \times \mathrm{w}}$ be nonsingular. $\mathfrak{B}$ is called $\Sigma$-dissipative if and only if $\int_{\mathbb{R}} w^{\top} \Sigma w d t \geq 0$ for all $w \in \mathfrak{B} \cap \mathfrak{D}\left(\mathbb{R}, \mathbb{R}^{\mathrm{w}}\right)$. $\mathfrak{B}$ is called strictly $\Sigma$-dissipative if and only if there exists $\varepsilon>0$ such that $\int_{\mathbb{R}} w^{\top} \Sigma w d t \geq \varepsilon \int_{\mathbb{R}} w^{\top} w d t$ for all $w \in \mathfrak{B} \cap \mathfrak{D}\left(\mathbb{R}, \mathbb{R}^{\mathrm{w}}\right) ; \mathfrak{B}$ is called strictly $\Sigma$-dissipative on $\mathbb{R}_{-}$if there exists $\varepsilon>0$ such that $\int_{\mathbb{R}_{-}} w^{\top} \Sigma w d t \geq \varepsilon \int_{\mathbb{R}_{-}} w^{\top} w d t$ for all $w \in \mathfrak{B} \cap$ $\mathfrak{D}\left(\mathbb{R}_{-}, \mathbb{R}^{\mathrm{w}}\right)$.

A subset $\mathfrak{B} \subset \mathfrak{L}_{2}^{\text {loc }}\left(\mathbb{R}, \mathbb{R}^{\mathrm{w}}\right)$ defines a linear differential system if there exists a polynomial matrix $R \in \mathbb{R}^{\mathrm{w} \times \mathrm{w}}[\xi]$ such that $\mathfrak{B}=\left\{w \in \mathfrak{L}_{2}^{\text {loc }}\left(\mathbb{R}, \mathbb{R}^{\mathrm{w}}\right) \mid R(d / d t) w=0\right\}$. We denote with $\mathfrak{L}^{\mathrm{w}}$ the set of linear differential systems with w external variables.

We call $\mathfrak{B} \in \mathfrak{L}^{\mathrm{w}}$ controllable if for all $w_{1}, w_{2} \in \mathfrak{B}$, there exists a $T \geq 0$ and a $w \in \mathfrak{B}$ such that $w(t)=w_{1}(t)$ for $t<0$ and $w(t+T)=w_{2}(t)$ for $t \geq 0$. We denote the controllable elements of $\mathfrak{L}^{\mathrm{w}}$ by $\mathfrak{L}_{\text {contr }}^{\mathrm{w}}$.

There are a number of important integer invariants associated with behaviors. The integer invariants associated with a linear differential behavior $\mathfrak{B}$ are the number of inputs, denoted $\mathrm{m}(\mathfrak{B})$, the number of outputs, denoted $\mathrm{p}(\mathfrak{B})$, and the dimension of a minimal state variable for $\mathfrak{B}$, equivalently called the McMillan degree of $\mathfrak{B}$ and denoted with $\mathrm{n}(\mathfrak{B})$.

Driving variable representations. Let $A \in \mathbb{R}^{\mathrm{n} \times \mathrm{n}}, B \in$ $\mathbb{R}^{\mathrm{n} \times \mathrm{m}}, C \in \mathbb{R}^{\mathrm{w} \times \mathrm{n}}, D \in \mathbb{R}^{\mathrm{w} \times \mathrm{m}}$ be constant real matrices. The equations

$$
\frac{d}{d t}=A x+B v, \quad w=C x+D v .
$$

represent the behavior

$$
\mathfrak{B}_{D V}(A, B, C, D):=\{(w, x, v) \mid(1) \text { holds }\} .
$$

This behavior is called the full behavior represented by (1). If we eliminate $x$ and $v$, then we get the external behavior defined by 


$$
\begin{aligned}
\mathfrak{B}_{D V}(A, B, C, D)_{\text {ext }}:= & \{w \mid \exists x, v \text { such that } \\
& \left.(w, x, v) \in \mathfrak{B}_{D V}(A, B, C, D)\right\} .
\end{aligned}
$$

It is well-known that for any given $\mathfrak{B} \in \mathfrak{L}^{\text {w }}$ there exist real constant matrices $A, B, C, D$ such that (see Weiland (1991))

$$
\mathfrak{B}=\mathfrak{B}_{D V}(A, B, C, D)_{\text {ext }} .
$$

In this case we call $\mathfrak{B}_{D V}(A, B, C, D)$ a driving variable representation of $\mathfrak{B}$. If $\mathrm{n}$ and $\mathrm{m}$ are minimal over all such driving variable representations, then we call $\mathfrak{B}_{D V}(A, B, C, D)$ a minimal driving variable representation. $\mathfrak{B}_{D V}(A, B, C, D)_{\text {ext }}$ can be shown to be controllable if and only if the pair $(A, B)$ is controllable.

\section{Characterization of dissipativity of behaviors in driving variable representations.}

Proposition 2. Let $\mathfrak{B} \in \mathfrak{L}_{\text {contr }}^{\text {w with } D V-r e p r e s e n t a t i o n ~}$ $\mathfrak{B}_{D V}(A, B, C, D)$ and $\Sigma=\Sigma^{\top} \in \mathbb{R}^{\mathrm{w} \times \mathrm{w}}$ nonsingular. Assume that $\mathrm{m}(\mathfrak{B})=\sigma_{+}(\Sigma)$, where $\mathrm{m}(\mathfrak{B})$ is the number of inputs of $\mathfrak{B}, \sigma_{+}(\Sigma)$ is the number of positive eigenvalues of $\Sigma$. Then the following statements are equivalent.

(1) $\mathfrak{B}$ is $\Sigma$-dissipative on $\mathbb{R}^{-}$.

(2) There exists a positive semidefinite solution $P=$ $P^{\top} \in \mathbb{R}^{\mathrm{n} \times \mathrm{n}}$ of the following LMI

$$
\left[\begin{array}{cc}
A^{\top} P+P A-C^{\top} \Sigma C & P B-C^{\top} \Sigma D \\
B^{\top} P-D^{\top} \Sigma C & -D^{\top} \Sigma D
\end{array}\right] \leq 0 .
$$

(3) All of solutions of LMI (2) are positive semidefinite, or equivalently, there exist $P_{-} \geq 0$ and $P_{+} \geq 0$ such that for any real solution $P$ of the LMI (2) we have $0 \leq P_{-} \leq P \leq P_{+}$.

(4) There exists a positive definite solution $0<P=$ $P^{\top} \in \mathbb{R}^{\mathrm{n} \times \mathrm{n}}$ of the $\operatorname{LMI}(2)$.

(5) All solutions of LMI (2) are positive definite, i.e. there exist $P_{-}>0$ and $P_{+}>0$ such that for any real solution $P$ of the LMI (2) we have $0<P_{-} \leq P \leq P_{+}$.

In addition, if $D^{\top} \Sigma D>0$ then any of the above statements is equivalent to

6) There exists a positive definite solution $0<P=$ $P^{\top} \in \mathbb{R}^{\mathrm{n} \times \mathrm{n}}$ of the following Algebraic Riccati Equation (ARE)

$$
\begin{aligned}
& A^{\top} P+P A-C^{\top} \Sigma C+ \\
& \left(P B-C^{\top} \Sigma D\right)\left(D^{\top} \Sigma D\right)^{-1}\left(B^{\top} P-D^{\top} \Sigma C\right)=0(3)
\end{aligned}
$$

7) All solutions of $\operatorname{ARE~(3)~are~positive~definite,~more-~}$ over the minimal solution $P_{-}$and the maximal solution $P_{+}$of the ARE (3) coincide with those of the LMI (2).

Proof. 1) $\Leftrightarrow 2$ ) is proved in (Willems et al., 1991, Theorem 8.4.5) and 2) $\Leftrightarrow 3) \Leftrightarrow 4) \Leftrightarrow 5) \Leftrightarrow 6) \Leftrightarrow 7)$ are proved in (Willems et al., 1998, Theorem 6.4).

Remark 3. We can also formulate Proposition 2 for the case that $\mathfrak{B}$ is $\Sigma$-dissipative on $\mathbb{R}^{+}$by replacing all of the words "positive" by "negative" in all of items 2) to 7).

Proposition 4. Let $\mathfrak{B} \in \mathfrak{L}_{\text {contr }}^{\mathrm{w}}$ with driving variable representation $\mathfrak{B}_{D V}(A, B, C, D)$ and $\Sigma=\Sigma^{\top} \in \mathbb{R}^{\mathrm{w} \times \mathrm{w}}$ nonsingular. Then the following statements are equivalent.

(1) $\mathfrak{B}$ is strictly $\Sigma$-dissipative on $\mathbb{R}^{-}$(or $\mathbb{R}^{+}$, respectively).
(2) $D^{\top} \Sigma D>0$ and there exists a positive definite antistabilizing (negative definite stabilizing) solution of $\operatorname{ARE}(3)$.

Proof. See Ha et al. (2006).

\section{2. $\Sigma$-NORMALIZATION}

The idea of $\Sigma$-normalization originates from the concept normalized coprime factorization in Hoffmann et al. (1996). In the following lemma we prove that the strictly dissipative behaviors that we are interested in allow a $\Sigma$ normalized representation.

Lemma 5. Assume that $\mathfrak{B} \in \mathfrak{L}_{\text {contr }}^{\text {w }}$ is strictly $\Sigma$-dissipative on $\mathbb{R}^{-}$and $\mathrm{m}(\mathfrak{B})=\sigma_{+}(\Sigma)$. Then there exist a minimal driving variable representation $\mathfrak{B}_{D V}(\bar{A}, \bar{B}, \bar{C}, \bar{D})$ of $\mathfrak{B}$ such that $\bar{A}$ is asymptotically stable and $\bar{G}^{\top}(-s) \Sigma \bar{G}(s)=$ $I$, where $\bar{G}(s):=\bar{D}+\bar{C}(s I-\bar{A})^{-1} \bar{B}$.

Proof. Let $\mathfrak{B}_{D V}(A, B, C, D)$ be a minimal driving variable representation of $\mathfrak{B}$. We first show that the ARE (3) has a stabilizing and antistabilizing solution $P_{-}$and $P_{+}$, respectively, and both of them are positive definite. Indeed, since $\mathfrak{B}$ is strictly $\Sigma$-dissipative on $\mathbb{R}^{-}$, it follows that (see Ha et al. (2006)) the following Hamiltonian matrix, denoted by $H$,

$$
\left[\begin{array}{cc}
A-B\left(D^{\top} \Sigma D\right)^{-1} D^{\top} \Sigma C & B\left(D^{\top} \Sigma D\right)^{-1} B^{\top} \\
C^{\top} \Sigma C-C^{\top} \Sigma D\left(D^{\top} \Sigma D\right)^{-1} D^{\top} \Sigma C & -\left(A-B\left(D^{\top} \Sigma D\right)^{-1} D^{\top} \Sigma C\right)^{\top}
\end{array}\right]
$$

has no eigenvalues on the imaginary axis. Therefore the smallest and largest solution $P_{-}$and $P_{+}$of the ARE (3) are stabilizing and antistabilizing, respectively. On the other hand, since $\mathfrak{B}$ is strictly $\Sigma$-dissipative on $\mathbb{R}^{-}$, $P_{+}>0$. Taking ito account the condition $\mathrm{m}(\mathfrak{B})=\sigma_{+}(\Sigma)$, by Proposition 2, we can conclude that all solutions of the ARE (3) are positive definite. Hence, $0<P_{-}<P_{+}$.

Set $F:=\left(D^{\top} \Sigma D\right)^{-1}\left(B^{\top} P_{-}-D^{\top} \Sigma C\right)$. Since $P_{-}$is a stabilizing solution, $A+B F$ is asymptotically stable. On the other hand, $D^{\top} \Sigma D>0$ and therefore there exists a nonsingular $R$ such that $D^{\top} \Sigma D=R^{\top} R$. Now by applying the feedback $v=F x+R^{-1} \bar{v}$ we get

$$
\begin{aligned}
& \dot{x}=\underbrace{(A+B F)}_{=: \bar{A}} x+\underbrace{B R^{-1}}_{=: \bar{B}} \bar{v}=\bar{A} x+\bar{B} \bar{v} \\
& w=\underbrace{(C+D F)}_{=: \bar{C}} x+\underbrace{D R^{-1}}_{=: \bar{D}} \bar{v}=\bar{C} x+\bar{D} \bar{v} .
\end{aligned}
$$

Since state feedback does not change the behavior $\mathfrak{B}$, this also generates a minimal representation $\mathfrak{B}_{D V}(\bar{A}, \bar{B}, \bar{C}, \bar{D})$ of $\mathfrak{B}$. It is not difficult to check that $\bar{G}^{\top}(-s) \Sigma \bar{G}(s)=I$, the required condition of lemma.

A representation satisfying the two conditions in Lemma 5 is called a $\Sigma$-normalized driving variable representation of $\mathfrak{B}$. $\Sigma$-normalized representations have some nice properties. This is elaborated in the following lemma.

Lemma 6. Assume that $\mathfrak{B} \in \mathfrak{L}_{\text {contr }}^{\text {w }}$ is strictly $\Sigma$-dissipative on $\mathbb{R}^{-}$and $\mathrm{m}(\mathfrak{B})=\sigma_{+}(\Sigma)$. Then the $\Sigma$-normalized driving variable representation $\mathfrak{B}_{D V}(\bar{A}, \bar{B}, \bar{C}, \bar{D})$ of $\mathfrak{B}$ constructed in the proof of Lemma 5 satisfies the following conditions:

(1) $\bar{A}^{\top} P_{-}+P_{-} \bar{A}-\bar{C}^{\top} \Sigma \bar{C}=0$,

(2) $\bar{B}^{\top} P_{-}-\bar{D}^{\top} \Sigma \bar{C}=0$,

(3) $\bar{D}^{\top} \Sigma \bar{D}=I$, 
Proof. The conditions 1, 2 and 3 can be verified by straightforward computation.

Remark 7. The equation in the first item in Lemma 6 looks similarly to Lyapunov equation defining the observability gramian. This leads to a notion of $\Sigma$-observability gramian: $M$ is called $\Sigma$-observability gramian which is the unique solution of equation $\bar{A}^{\top} M+M \bar{A}-\bar{C}^{\top} \Sigma \bar{C}=0$. When $\Sigma=I, \Sigma$-observability gramian concides with observability gramian.

The above lemma suggests that the controllability and $\Sigma$-observability gramians are related to the maximal and minimal solutions of ARE (3).

Lemma 8. Assume that $\mathfrak{B} \in \mathfrak{L}_{\text {contr }}^{\text {w }}$ is strictly $\Sigma$-dissipative on $\mathbb{R}^{-}$and $\mathrm{m}(\mathfrak{B})=\sigma_{+}(\Sigma)$. Assume that $\mathfrak{B}_{D V}(\bar{A}, \bar{B}, \bar{C}, \bar{D})$ is the $\Sigma$-normalized driving variable representation of $\mathfrak{B}$ contructed in the proof of Lemma 5. Then

(1) $M=P_{-}$,

(2) $W=\left(P_{+}-P_{-}\right)^{-1}$,

where $M$ is $\Sigma$-observability gramian, (the unique solution of $\bar{A}^{\top} M+M \bar{A}-\bar{C}^{\top} \Sigma \bar{C}=0$ ), and $W$ is controllability gramian, (the unique solution of $\bar{A} W+W \bar{A}^{\top}+\bar{B} \bar{B}^{\top}=0$ ).

Proof. 1) follows from Lemma 6.

2) Using facts $\bar{A}^{\top} P_{-}+P_{-} \bar{A}=\bar{C}^{\top} \Sigma \bar{C}, \bar{B}^{\top} P_{-}=\bar{D}^{\top} \Sigma \bar{C}$ and $\bar{D}^{\top} \Sigma \bar{D}=I$ from Lemma 6 , we get

$$
\begin{aligned}
& \left(P_{+}-P_{-}\right) \bar{A}+\bar{A}^{\top}\left(P_{+}-P_{-}\right) \\
& +\left(P_{+}-P_{-}\right) \bar{B} \bar{B}^{\top}\left(P_{+}-P_{-}\right) \\
= & P_{+} \bar{A}+\bar{A}^{\top} P_{+}-P_{-} \bar{A}-\bar{A}^{\top} P_{-} \\
& +\left(P_{+} \bar{B}-P_{-} \bar{B}\right)\left(\bar{B}^{\top} P_{+}-\bar{B}^{\top} P_{-}\right) \\
= & P_{+} \bar{A}+\bar{A}^{\top} P_{+}-\bar{C}^{\top} \Sigma \bar{C} \\
& +\left(P_{+} \bar{B}-\bar{C}^{\top} \Sigma \bar{D}\right)\left(\bar{D}^{\top} \Sigma \bar{D}\right)^{-1}\left(\bar{B}^{\top} P_{+}-\bar{D}^{\top} \Sigma \bar{C}\right) \\
= & 0, \quad\left(\text { since } P_{+} \text {is solution of } \mathrm{ARE}(3)\right) .
\end{aligned}
$$

Therefore,

$$
\bar{A}\left(P_{+}-P_{-}\right)^{-1}+\left(P_{+}-P_{-}\right)^{-1} \bar{A}^{\top}+\bar{B} \bar{B}^{\top}=0,
$$

Hence $\left(P_{+}-P_{-}\right)^{-1}=W$, the unique solution of the Lyapunov equation $\bar{A} W+W \bar{A}^{\top}+\bar{B} \bar{B}^{\top}=0$.

\section{3. $\Sigma$-BALANCED TRUNCATION}

Assume that $\mathfrak{B} \in \mathfrak{L}_{\text {contr }}^{\text {w }}$ is strictly $\Sigma$-dissipative on $\mathbb{R}^{-}$ and $\mathrm{m}(\mathfrak{B})=\sigma_{+}(\Sigma)$. Let $\mathfrak{B}_{D V}(A, B, C, D)$ be a minimal driving variable representation of $\mathfrak{B}$ and let $P_{-}$and $P_{+}$ be the minimal and maximal solutions of the ARE (3) respectively. Then the eigenvalues of $P_{-} P_{+}^{-1}$ are real, positive, strictly less than one and similarity invariants with respect to state transformations. Their positive square roots are called the $\Sigma$-characteristic values of $\mathfrak{B}$. Let $1>\sigma_{1}^{2} \geq \sigma_{2}^{2} \geq \cdots \geq \sigma_{n}^{2}>0$ denote the $n$ eigenvalues of $P_{-} P_{+}^{-1}$. Then there exists a similarity transformation which transforms both $P_{+}^{-1}$ and $P_{-}$to the diagonal form $P_{+}^{-1}=P_{-}=\operatorname{diag}\left(\sigma_{1}, \sigma_{2}, \ldots, \sigma_{n}\right)=: \Pi$. The new system is then said to be in $\Sigma$-balanced coordinates.

Pick $k<n$ such that $\sigma_{k}>\sigma_{k+1}$ and partition $\Pi$ into

$$
\Pi=\left[\begin{array}{cc}
\Pi_{1} & 0 \\
0 & \Pi_{2}
\end{array}\right]
$$

where $\Pi_{1}=\operatorname{diag}\left(\sigma_{1}, \ldots, \sigma_{k}\right), \Pi_{2}=\operatorname{diag}\left(\sigma_{k+1}, \ldots, \sigma_{n}\right)$. Partition $A, B$ and $C$ comformably with the partitioning of $\Pi$ :

$$
A=\left[\begin{array}{ll}
A_{11} & A_{12} \\
A_{21} & A_{22}
\end{array}\right], B=\left[\begin{array}{l}
B_{1} \\
B_{2}
\end{array}\right], C=\left[\begin{array}{ll}
C_{1} & C_{2}
\end{array}\right] .
$$

By truncating and retaining the first $k$ states of the original model, we can then define a reduced order behavior $\hat{\mathfrak{B}}$ by $\hat{\mathfrak{B}}:=\mathfrak{B}_{D V}\left(A_{11}, B_{1}, C_{1}, D\right)_{\text {ext }}$. In the following, we investigate the balanced truncation method for $\Sigma$-normalized representations. Starting with a $\Sigma$-normalized driving variable representation, transforming into $\Sigma$-balanced coordinates results in a $\Sigma$-normalized driving variable representation as well.

Theorem 9. Assume that $\mathfrak{B} \in \mathfrak{L}_{\text {contr }}^{\mathrm{w}}$ is strictly $\Sigma$ dissipative on $\mathbb{R}^{-}$and $\mathrm{m}(\mathfrak{B})=\sigma_{+}(\Sigma)$. Let $\mathfrak{B}_{D V}(A, B, C, D)$ be a $\Sigma$-normalized driving variable representation of $\mathfrak{B}$. Then after balancing, we obtain $\mathfrak{B}_{D V}(\bar{A}, \bar{B}, \bar{C}, \bar{D})$ in $\Sigma$ balanced coordinates and this is also a $\Sigma$-normalized driving variable representation of $\mathfrak{B}$.

Proof. This result follows from the fact that the transfer matrix associated with the driving variable representation does not change under coordinate transformation.

From now on, for a given $\mathfrak{B}$, we will work with a $\Sigma$-normalized minimal driving variable representation $\mathfrak{B}_{D V}(\bar{A}, \bar{B}, \bar{C}, \bar{D})$ which is in $\Sigma$-balanced coordinates, that is in these coordinates $P_{+}^{-1}=P_{-}=\operatorname{diag}\left(\sigma_{1}, \sigma_{2}, \ldots, \sigma_{n}\right)=$ $\Pi$.

Lemma 10. Assume that $\mathfrak{B}_{D V}(\bar{A}, \bar{B}, \bar{C}, \bar{D})$ is a $\Sigma$-normalized minimal driving variable representation of $\mathfrak{B}$. In $\Sigma$ balanced coordinates, the $\Sigma$-observability gramian $M$ and controllability gramian $W$ are given by

(1) $M=\Pi=\operatorname{diag}\left(\sigma_{1}, \ldots, \sigma_{n}\right)$,

(2) $W=\left(\Pi^{-1}-\Pi\right)^{-1}=\operatorname{diag}\left(\frac{\sigma_{1}}{1-\sigma_{1}^{2}}, \ldots, \frac{\sigma_{n}}{1-\sigma_{n}^{2}}\right)$.

Proof. This is a consequence of Lemma 8.

Looking at Lemma 10 from a different point of view, $\Sigma$-balanced coordinates can be viewed as coordinates in which the Lyapunov equations have solutions in diagonal form. Indeed, we have

$$
\begin{gathered}
\bar{A}^{\top} \Pi+\Pi \bar{A}+\bar{C}^{\top} \Sigma \bar{C}=0, \\
\bar{A}\left(\Pi^{-1}-\Pi\right)^{-1}+\left(\Pi^{-1}-\Pi\right)^{-1} \bar{A}^{\top}+\bar{B} \bar{B}^{\top}=0 .
\end{gathered}
$$

Next, we take a look at the reduced order behavior $\hat{\mathfrak{B}}=$ $\mathfrak{B}_{D V}\left(\bar{A}_{11}, \bar{B}_{1}, \bar{C}_{1}, \bar{D}\right)_{\text {ext }}$ obtained by truncation of the driving variable representation $\mathfrak{B}_{D V}(\bar{A}, \bar{B}, \bar{C}, \bar{D})$. Our aim is to be able to conclude that dissipativeness is preserved after reduction.

Theorem 11. Assume that $\mathfrak{B} \in \mathfrak{L}_{\text {contr }}^{\mathrm{w}}$ is strictly $\Sigma$ dissipative on $\mathbb{R}^{-}$and $\mathrm{m}(\mathfrak{B})=\sigma_{+}(\Sigma)$. Let $\mathfrak{B}_{D V}(\bar{A}, \bar{B}, \bar{C}, \bar{D})$ be a $\Sigma$-normalized driving variable representation of $\mathfrak{B}$, in $\Sigma$-balanced coordinates. Then $\sigma\left(\bar{A}_{11}\right) \subset \mathbb{C}^{-} \cup i \mathbb{R}$. We also have: $\sigma\left(\bar{A}_{11}\right) \subset \mathbb{C}^{-}$if and only if $\left(\bar{A}_{11}, \bar{B}_{1}\right)$ is controllable.

Proof. Since $\mathfrak{B}_{D V}(\bar{A}, \bar{B}, \bar{C}, \bar{D})$ is a $\Sigma$-normalized driving variable representation of $\mathfrak{B}$ and is in $\Sigma$-balanced coordinates, we have

$$
\bar{A}\left(\Pi^{-1}-\Pi\right)^{-1}+\left(\Pi^{-1}-\Pi\right)^{-1} \bar{A}^{\top}+\bar{B} \bar{B}^{\top}=0 .
$$

It follows that

$$
\bar{A}_{11} \Theta_{11}+\Theta_{11} \bar{A}_{11}^{\top}+\bar{B}_{1} \bar{B}_{1}^{\top}=0,
$$


where $\Theta_{11}:=\left(\Pi_{11}^{-1}-\Pi_{11}\right)^{-1}$. Let $\lambda$ be an eigenvalue of $\bar{A}_{11}$ and $v \neq 0$ be a corresponding left eigenvector, i.e. $v^{*} \bar{A}_{11}=v^{*} \lambda$. Premultiplying the above equation with $v^{*}$ and postmultiplying by $v$ we obtain

$$
2 \operatorname{Re}(\lambda)\left(v^{*} \Theta_{11} v\right)+v^{*} \bar{B}_{1} \bar{B}_{1}^{\top} v=0 .
$$

Since $v^{*} \bar{B}_{1} \bar{B}_{1}^{\top} v \geq 0$ and $v^{*} \Theta_{11} v>0$, it follows that $\operatorname{Re}(\lambda) \leq 0$. We will show that $\operatorname{Re}(\lambda)<0$ if and only if $\left(\bar{A}_{11}, \bar{B}_{1}\right)$ is controllable. In fact, if $\operatorname{Re}(\lambda)=0$, then $v^{*} \bar{B}_{1} \bar{B}_{1}^{\top} v=0$, or $v^{*} \bar{B}_{1}=0$. Therefore $v^{*}\left(\bar{A}_{11}-\lambda I \bar{B}_{1}\right)=$ 0 , which contradicts controllability of $\left(\bar{A}_{11}, \bar{B}_{1}\right)$. Conversely, assume $\sigma\left(\bar{A}_{11}\right) \subset \mathbb{C}^{-}$but $\left(\bar{A}_{11}, \bar{B}_{1}\right)$ is not controllable. Then there exists $v \neq 0$ and an eigenvalue $\lambda$ of $\bar{A}_{11}$ such that $v^{*} \bar{A}_{11}=\lambda v^{*}$ and $v^{*} \bar{B}_{1}=0$. In the same way as above we obtain $2 \operatorname{Re}(\lambda) v^{*} \Theta_{11} v=v^{*} \bar{B}_{1} \bar{B}_{1}^{\top} v=0$, which yields a contradiction. We conclude that $\left(\bar{A}_{11}, \bar{B}_{1}\right)$ is controllable.

Next, we claim that balanced truncation preserves $\Sigma$ normalized representation.

Theorem 12. Assume that $\mathfrak{B} \in \mathfrak{L}_{\text {contr }}^{\mathrm{w}}$ is strictly $\Sigma$ dissipative on $\mathbb{R}^{-}$and $\mathrm{m}(\mathfrak{B})=\sigma_{+}(\Sigma)$. Let $\mathfrak{B}_{D V}(\bar{A}, \bar{B}, \bar{C}, \bar{D})$ be a $\Sigma$-normalized driving variable representation of $\mathfrak{B}$, in $\Sigma$-balanced coordinates. Assume that $\sigma\left(\bar{A}_{11}\right) \subset \mathbb{C}^{-}$. Then the balanced truncation $\mathfrak{B}_{D V}\left(\bar{A}_{11}, \bar{B}_{1}, \bar{C}_{1}, \bar{D}\right)$ is a $\Sigma$-normalized representation of $\hat{\mathfrak{B}}$, and is in balanced coordinates.

Proof. It is easily checked that $\bar{G}_{11}^{\top}(-s) \Sigma \bar{G}_{11}(s)=I$, where $\bar{G}_{11}(s):=\bar{D}+\bar{C}_{1}\left(s I-\bar{A}_{11}\right)^{-1} \bar{B}_{1}$.

It turns out that balanced truncation also preserves strict dissipativeness of systems.

Theorem 13. Assume that $\mathfrak{B} \in \mathfrak{L}_{\text {contr }}^{\mathrm{w}}$ is strictly $\Sigma$ dissipative on $\mathbb{R}^{-}$and $\mathrm{m}(\mathfrak{B})=\sigma_{+}(\Sigma)$. Assume that $\sigma\left(\bar{A}_{11}\right) \subset \mathbb{C}^{-}$. Then the reduced order behavior $\hat{\mathfrak{B}}$ obtained above is controllable, and strictly $\Sigma$-dissipative on $\mathbb{R}^{-}$.

Proof. By Proposition $4, \mathfrak{B}$ is $\Sigma$-strictly dissipative on $\mathbb{R}^{-}$ if and only if $D^{\top} \Sigma D>0$ and the antistabilizing solution $P_{+}$of ARE (3) is positive definite. Strict dissipativity also implies that the Hamiltonian matrix $H$

$$
\left[\begin{array}{cc}
A-B\left(D^{\top} \Sigma D\right)^{-1} D^{\top} \Sigma C & B\left(D^{\top} \Sigma D\right)^{-1} B^{\top} \\
C^{\top} \Sigma C-C^{\top} \Sigma D\left(D^{\top} \Sigma D\right)^{-1} D^{\top} \Sigma C & -\left(A-B\left(D^{\top} \Sigma D\right)^{-1} D^{\top} \Sigma C\right)^{\top}
\end{array}\right]
$$

has no eigenvalues on the imaginary axis. Therefore there exist stabilizing and antistabilizing solutions $P_{-}$and $P_{+}$. It is true that $\sigma\left(A+B\left(D^{\top} \Sigma D\right)^{-1}\left(B^{\top} P_{-}-D^{\top} \Sigma C\right)\right)=$ $\sigma_{-}(H)$ and $\sigma\left(A+B\left(D^{\top} \Sigma D\right)^{-1}\left(B^{\top} P_{+}-D^{\top} \Sigma C\right)\right)=$ $\sigma_{+}(H)$.

Without loss of generality, we assume that $\mathfrak{B}$ is in $\Sigma$ normalized driving variable representation $\mathfrak{B}_{D V}(A, B, C, D)$ Then after balancing, by Theorem 9 we obtain that $\mathfrak{B}_{D V}(\bar{A}, \bar{B}, \bar{C}, \bar{D})$ is in $\Sigma$-balanced coordinates and also a $\Sigma$-normalized driving variable representation of $\mathfrak{B}$. The reduced model $\mathfrak{B}_{D V}\left(\bar{A}_{11}, \bar{B}_{1}, \bar{C}_{1}, \bar{D}\right)$ obtained after truncation has $\bar{A}_{11}$ aymptotically stable. Therefore the reduced ARE:

$$
\begin{array}{r}
\bar{A}_{11}^{\top} \hat{P}+\hat{P} \bar{A}_{11}-\bar{C}_{1}^{\top} \Sigma \bar{C}_{1}+\left(\hat{P} \bar{B}_{1}-\bar{C}_{1}^{\top} \Sigma \bar{D}\right) \\
\left(\bar{D}^{\top} \Sigma \bar{D}\right)^{-1}\left(\bar{B}_{1}^{\top} \hat{P}-\bar{D}^{\top} \Sigma \bar{C}_{1}\right)=0
\end{array}
$$

has the stabilizing solution $\Pi_{11}$. This implies that this ARE also has an antistablizing solution, say $\hat{P}_{+}$. Since
$\hat{P}_{+}>\Pi_{11}>0$, by Proposition 4 again, $\hat{\mathfrak{B}}$ is strictly $\Sigma$ dissipative on $\mathbb{R}^{-}$.

In order to provide a physical meaning of balanced truncation, let us introduce concepts of available storage and required supply of the dissipative system. $V_{a v}\left(x_{0}\right)$ is available storage, or the maximum amount of energy that can be extracted from the system over any possible trajectories which has initial state $x_{0}$ at time zero. In (Willems et al., 1991, Theorem 8.4.2), $V_{a v}\left(x_{0}\right)=x_{0}^{\top} P_{-} x_{0}$, hence the importance of each components of the state $x_{0}$ (importance in the sense that: we want to extract as much as possible energy from each components of $x_{0}$ ) is proportional to $P_{-}$. This point of view suggests that we should re-order the components of the state in the way proportional to $P_{-}$. On the other hand, in (Willems et al., 1991, Theorem 8.4.2), $V_{\text {req }}\left(x_{0}\right)=x_{0}^{\top} P_{+} x_{0}$ is required supply, or the minimum amount energy that has to be supplied to the system in order to drive the system to state $x_{0}$ at time zero over any possible trajectories. We again introduce new concept of importance of each components (importance in the sense that: we want to reduce as much as possible energy that bring the system to each components of $x_{0}$ ). In this case, the importance of each components is inversely proportional to $P_{+}$, hence we should re-order the components of the state in the way inversely proportional to $P_{+}$. The idea of balanced coordinates is that to re-order the components of the state which meet both requirements, that means we bring to the new system such that the first component require the least energy to supply but has the most ernergy to extract, the second component has more energy to supply and less ernergy to extract than the first one, the third can be compared with the second and so on. Likewise, the first component is the most inportant, the second component is less important than the first one and so on... Since we want to keep the important components, the tail $n-k$ components of the state have to be truncated.

\section{ERROR BOUND}

\subsection{A one-step error bound}

For driving variable representations, $G(s)=D+C(s I-$ $A)^{-1} B$ is the transfer function from the driving variable $v$ to the manifest variable $w$. We will now investigate $\left[G(-s)^{\top}-\hat{G}(-s)^{\top}\right] \Sigma[G(s)-\hat{G}(s)]$, where $\hat{G}(s)=D+$ $C_{1}\left(s I-A_{11}\right)^{-1} B_{1}$ is the transfer function from the driving variable to the manifest variable in the driving variable representation of the reduced order behavior.

The following result gives an estimate of a one-step error bound. One-step means that we truncate only one state variable, i.e., the McMillan degree of the to be reduced model is reduced by one.

Theorem 14. Assume that $\mathfrak{B}_{D V}(A, B, C, D)$ is a $\Sigma$-normalized, $\Sigma$-balanced driving variable representation of $\mathfrak{B}$. Let $\hat{G}(s)$ be the transfer matrix from the driving variable to the manifest variable of the reduced order behavior obtained from the orginial one by truncating one state variable. Then for all $\omega \in \mathbb{R}$ we have:

$$
-[G(-i \omega)-\hat{G}(-i \omega)]^{\top} \Sigma[G(i \omega)-\hat{G}(i \omega)] \leq \frac{4 \sigma_{n}^{2}}{1-\sigma_{n}^{2}} I .
$$


Proof. We introduce the "error system", $E(s):=G(s)-$ $\hat{G}(s)$. By straightforward computation, $E(s)=C(s)(s I-$ $A(s))^{-1} B(s)=\left[\begin{array}{c|c}A(s) & B(s) \\ \hline C(s) & 0\end{array}\right]$, where

$$
\begin{aligned}
& A(s):=A_{22}+A_{21}\left(s I-A_{11}\right)^{-1} A_{12}, \\
& B(s):=B_{2}+A_{21}\left(s I-A_{11}\right)^{-1} B_{1}, \\
& C(s):=C_{2}+C_{1}\left(s I-A_{11}\right)^{-1} A_{12} .
\end{aligned}
$$

Assume that $M$ and $W$ are partitioned comformably with $\Pi$ as

$$
M=\left[\begin{array}{cc}
M_{1} & 0 \\
0 & M_{2}
\end{array}\right], W=\left[\begin{array}{cc}
W_{1} & 0 \\
0 & W_{2}
\end{array}\right]
$$

It is not difficult to verify that

$$
\begin{aligned}
A(-s)^{\top} M_{2}+M_{2} A(s)-C(-s)^{\top} \Sigma C(s) & =0, \\
A(s) W_{2}+W_{2} A(-s)^{\top}+B(s) B\left(-s^{\top}\right) & =0 .
\end{aligned}
$$

In our case, $M_{2}=\sigma_{n}, W_{2}=\frac{\sigma_{n}}{1-\sigma_{n}^{2}}$ since we truncate only one state variable. Transforming equation (5) we get

$$
\begin{aligned}
& \sigma_{n} A(-s)^{\top}+\sigma_{n} A(s)-C(-s)^{\top} \Sigma C(s)=0 \\
\Leftrightarrow & \sigma_{n}\left[-s I-A(-s)^{\top}\right]+\sigma_{n}[s I-A(s)] \\
& =-C(-s)^{\top} \Sigma C(s) \\
\Leftrightarrow & \sigma_{n}\left[-s I-A(-s)^{\top}\right]^{-1}+\sigma_{n}[s I-A(s)]^{-1}= \\
& -\left[-s I-A(-s)^{\top}\right]^{-1} C(-s)^{\top} \Sigma C(s)[s I-A(s)]^{-1} \\
\Leftrightarrow & \sigma_{n} B(-s)^{\top}\left[-s I-A(-s)^{\top}\right]^{-1} B(s)+ \\
& \sigma_{n} B(-s)^{\top}[s I-A(s)]^{-1} B(s)= \\
& -B(-s)^{\top}\left[-s I-A(-s)^{\top}\right]^{-1} C(-s)^{\top} \Sigma \\
& C(s)[s I-A(s)]^{-1} B(s) \\
\Leftrightarrow & \sigma_{n} R(-s)^{\top}+\sigma_{n} R(s)=-E(-s)^{\top} \Sigma E(s),
\end{aligned}
$$

where $R(s):=B(-s)^{\top}[s I-A(s)]^{-1} B(s)$ and $E(s)=$ $C(s)(s I-A(s))^{-1} B(s)$ as above. Similary, with equation (6) we get

$$
\frac{\sigma_{n}}{1-\sigma_{n}^{2}} R(-s)^{\top}+\frac{\sigma_{n}}{1-\sigma_{n}^{2}} R(s)=R(-s)^{\top} R(s) .
$$

Now, from (7) and (8)

$$
\begin{aligned}
& -E(-s)^{\top} \Sigma E(s)=\sigma_{n} R(-s)^{\top}+\sigma_{n} R(s) \\
= & 2 \sigma_{n} R(-s)^{\top}+2 \sigma_{n} R(s)-\left(1-\sigma_{n}^{2}\right) R(-s)^{\top} R(s) \\
= & \frac{4 \sigma_{n}^{2}}{1-\sigma_{n}^{2}} I-\left[\sqrt{1-\sigma_{n}^{2}} R(-s)^{\top}-\frac{2 \sigma_{n}}{\sqrt{1-\sigma_{n}^{2}}} I\right] \\
& {\left[\sqrt{1-\sigma_{n}^{2}} R(s)-\frac{2 \sigma_{n}}{\sqrt{1-\sigma_{n}^{2}}} I\right] . }
\end{aligned}
$$

Replacing $s=i \omega$, we get

$$
\begin{aligned}
& -E(-i \omega)^{\top} \Sigma E(i \omega) \\
= & \frac{4 \sigma_{n}^{2}}{1-\sigma_{n}^{2}} I-\left[\sqrt{1-\sigma_{n}^{2}} R(i \omega)-\frac{2 \sigma_{n}}{\sqrt{1-\sigma_{n}^{2}}} I\right]^{\top} \\
& {\left[\sqrt{1-\sigma_{n}^{2}} R(i \omega)-\frac{2 \sigma_{n}}{\sqrt{1-\sigma_{n}^{2}}} I\right] } \\
\leq & \frac{4 \sigma_{n}^{2}}{1-\sigma_{n}^{2}} I .
\end{aligned}
$$

\section{APPLICATIONS}

In this section, we consider the bounded real balancing of input/state/output systems. Consider the equations

$$
\left\{\begin{array}{l}
\dot{x}=\bar{A} x+\bar{B} u \\
y=\bar{C} x+\bar{D} u
\end{array}\right.
$$

With $(A, B)$ controllable and $(C, A)$ observable. These equations represent the input-state-output system

$\mathfrak{B}_{i / s / o}:=\{(u, y, x) \mid$ there exists $x$ such that (9) holds $\}$

In turn, the external behavior of this input-state-output system is the input-output system $\mathfrak{B}$ given by

$$
\mathfrak{B}=\{(u, y) \mid \text { there exists } x \text { such that (9) holds }\}
$$

$\mathfrak{B}$ is said to be strictly bounded real if it is strictly $\Sigma$ dissipative on $\mathbb{R}^{-}$, with $\Sigma$ given by

$$
\Sigma=\left(\begin{array}{cc}
I_{m} & 0 \\
0 & -I_{p}
\end{array}\right) \text {. }
$$

In Opdenacker et al. (1988), a bounded real balanced truncation scheme is introduced and an $L^{\infty}$ error bound is provided. Let $H(s):=\bar{D}+\bar{C}(s I-\bar{A})^{-1} \bar{B}$ be the transfer function from input $u$ to output $y$ of original system and $\hat{H}(s)$ be the transfer function of the reduced order model. In Opdenacker et al. (1988) the following error bound formula is derived:

$$
\|H(s)-\hat{H}(s)\|_{\infty} \leq 2 \sum_{i=k+1}^{n} \sigma_{i},
$$

where $\sigma_{i}, i=1 \ldots n$ are the $\Sigma$-characteristic values of $\mathfrak{B}$.

Now we use the theory of $\Sigma$-balancing developed in this paper to investigate this model from a different angle. Note that $\mathfrak{B}$ also has a driving variable representation $\mathfrak{B}_{D V}\left(\bar{A}, \bar{B},\left[\begin{array}{c}0 \\ \bar{C}\end{array}\right],\left[\begin{array}{c}I \\ \bar{D}\end{array}\right]\right)$.

We will perform the three following steps: 1) $\Sigma$-normalization, 2) $\Sigma$-balancing and truncation, 3) investigation of the error bound.

1) $\Sigma$-normalization: Apply the feedback $v=F x+$ $R^{-1} \bar{v}$, where $F:=\left(I-\bar{D}^{\top} \bar{D}\right)^{-1}\left(\bar{B} P_{-}+\bar{D}^{\top} \bar{C}\right)$ and $I-\bar{D}^{\top} \bar{D}=R^{\top} R$. Then the new, $\Sigma$-normalized, driving variable representation $\mathfrak{B}_{D V}(A, B, C, D)$ of $\mathfrak{B}$ is given by:

$$
\begin{aligned}
A & :=\bar{A}+\bar{B} F=\bar{A}+\bar{B}\left(I-\bar{D}^{\top} \bar{D}\right)^{-1}\left(\bar{B} P_{-}+\bar{D}^{\top} \bar{C}\right), \\
B & :=\bar{B} R^{-1}, \\
C & :=\left[\begin{array}{c}
0 \\
\bar{C}
\end{array}\right]+\left[\begin{array}{c}
I \\
\bar{D}
\end{array}\right] F \\
& =\left[\begin{array}{c}
\left(I-\bar{D}^{\top} \bar{D}\right)^{-1}\left(\bar{B} P_{-}+\bar{D}^{\top} \bar{C}\right) \\
\bar{C}+\bar{D}\left(I-\bar{D}^{\top} \bar{D}\right)^{-1}\left(\bar{B} P_{-}+\bar{D}^{\top} \bar{C}\right)
\end{array}\right], \\
D & :=\left[\begin{array}{c}
I \\
\bar{D}
\end{array}\right] R^{-1}=\left[\begin{array}{c}
R^{-1} \\
\bar{D} R^{-1}
\end{array}\right] .
\end{aligned}
$$

2) $\Sigma$-balancing and truncation: Using the algorithm shown in Section 3, we obtain the reduced order behavior $\hat{\mathfrak{B}}$ which again strictly bounded real (by Theorem 13). The driving variable representation $\mathfrak{B}_{D V}\left(A_{11}, B_{1}, C_{1}, D\right)$ of $\hat{\mathfrak{B}}$ is $\Sigma$ normalized (by Theorem 12). 
3) Investigating the error bound: Consider the $\Sigma$ normalized driving variable representation $\mathfrak{B}_{D V}(A, B, C, D)$ for $\mathfrak{B}$ obtained in step 1$)$, and let $\mathfrak{B}_{D V}\left(A_{11}, B_{1}, C_{1}, D\right)$ be the driving variable representation of the reduced order system obtained in 2). Denote by $G(s)$ and $\hat{G}(s)$ the transfer matrix from driving variable to manifest variable of $\mathfrak{B}_{D V}(A, B, C, D)$ and $\mathfrak{B}_{D V}\left(A_{11}, B_{1}, C_{1}, D\right)$, respectively. Partition

$$
G(s)=\left(\begin{array}{l}
G_{1}(s) \\
G_{2}(s)
\end{array}\right)
$$

where $G_{1}(s)$ is the transfer matrix from $v$ to $u$ and $G_{2}(s)$ the transfer matrix from $v$ to $y$. Likewise, let $\hat{G}_{1}(s)$ and $\hat{G}_{2}(s)$ be the transfer matrices from the driving variable to $\hat{u}$ and $\hat{y}$, respectively. Assume now that we truncate one step. Theorem 14 says that

$$
\begin{aligned}
& -[G(-i \omega)-\hat{G}(-i \omega)]^{\top} \Sigma[G(i \omega)-\hat{G}(i \omega)] \\
= & -\left(\left(\begin{array}{c}
G_{1}(-i \omega) \\
G_{2}(-i \omega)
\end{array}\right)-\left(\begin{array}{c}
\hat{G}_{1}(-i \omega) \\
\hat{G}_{2}(-i \omega)
\end{array}\right)\right)^{\top} \\
& \left(\begin{array}{cc}
I_{m} & 0 \\
0 & -I_{p}
\end{array}\right)\left(\left(\begin{array}{c}
G_{1}(i \omega) \\
G_{2}(i \omega)
\end{array}\right)-\left(\begin{array}{c}
\hat{G}_{1}(i \omega) \\
\hat{G}_{2}(i \omega)
\end{array}\right)\right) \\
= & -\left[G_{1}(-i \omega)-\hat{G}_{1}(-i \omega)\right]^{\top}\left[G_{1}(i \omega)-\hat{G}_{1}(i \omega)\right] \\
& +\left[G_{2}(-i \omega)-\hat{G}_{2}(-i \omega)\right]^{\top}\left[G_{2}(i \omega)-\hat{G}_{2}(i \omega)\right] \\
\leq & \frac{4 \sigma_{n}^{2}}{1-\sigma_{n}^{2}} I .
\end{aligned}
$$

This implies

$$
\begin{aligned}
& {\left[G_{2}(-i \omega)-\hat{G}_{2}(-i \omega)\right]^{\top}\left[G_{2}(i \omega)-\hat{G}_{2}(i \omega)\right] } \\
\leq & \frac{4 \sigma_{n}^{2}}{1-\sigma_{n}^{2}} I+\left[G_{1}(-i \omega)-\hat{G}_{1}(-i \omega)\right]^{\top}\left[G_{1}(i \omega)-\hat{G}_{1}(i \omega)\right] .
\end{aligned}
$$

This implies that if we 'drive' both $\mathfrak{B}$ and $\hat{\mathfrak{B}}$ with the same driving variable trajectory $v \in \mathfrak{L}_{2}(\mathbb{R})$, with corresponding input trajectories $u, \hat{u} \in \mathfrak{L}_{2}(\mathbb{R})$ and $y, \hat{y} \in \mathfrak{L}_{2}(\mathbb{R})$ for $\mathfrak{B}$ and $\hat{\mathfrak{B}}$, respectively, then we have

$$
\|y-\hat{y}\|_{2}^{2} \leq \frac{4 \sigma_{n}^{2}}{1-\sigma_{n}^{2}}\|v\|_{2}^{2}+\|u-\hat{u}\|_{2}^{2},
$$

which means that the squared $\mathfrak{L}_{2}$-norm of the difference of the outputs of the two systems does not exceed the sum of the squared $\mathfrak{L}_{2}$-norm of the difference of the inputs and a term depending on $\sigma_{n}$ and the squared $\mathfrak{L}_{2}$-norm of the driving variable. If the driving variable $v$ is taken such that the two inputs $u$ and $\hat{u}$ are the same, then we obtain

$$
\|y-\hat{y}\|_{2}^{2} \leq \frac{4 \sigma_{n}^{2}}{1-\sigma_{n}^{2}}\|v\|_{2}^{2}
$$

This formula is valids for one-step truncation only. It turns out that in the case that we truncate $k$ state variables, using the triangular inequality we get

$$
\|y-\hat{y}\|_{2}^{2} \leq \sum_{i=k+1}^{n} \frac{4 \sigma_{i}^{2}}{1-\sigma_{i}^{2}}\|v\|_{2}^{2} .
$$

Here, the driving variable $v$ should be such that the inputs of al $i$ th order truncations $(i=0,1,2, \ldots, k)$ are the same.
(1) A new balanced truncation method based on normalized representation has been presented. Dissipativeness is preserved after reduction and an one-step error bound formula is discussed.

(2) In the future we intend to find algorithms to reduce the model from output nulling representation to output nulling representation.

\section{REFERENCES}

A.C. Antoulas, Approximation of large-scale dynamical systems, SIAM Press, Philadelphia, 2004.

Xin Chen, John T. Wen, "Positive realness preserving model reduction with $H_{\infty}$ norm error bounds," IEEE Trans. Circuits Systems, 42, 1995, no. 1, 23-29.

H.B. Minh, P. Rapisarda, and H.L. Trentelman, "Model Reduction by Retention of Stationary Trajectories", Proceeding of the 17th International Symposium on Mathematical Theory of Networks and Systems, Kyoto, Japan, pp. 1639-1642, July 24-28, 2006.

Hoffmann, J. W., Fuhrmann, P. A. "On balanced realizations of bounded real and positive real functions", Linear Algebra Appl., 245, 1996, 107-146.

Philippe C. Opdenacker, Edmond A. Jonckheere, "A contraction mapping preserving balanced reduction scheme and its infinity norm error bounds", IEEE Trans. Circuits and Systems, 35, 1988, no. 2, 184-189.

P. Rapisarda, J.C. Willems, "Balanced state representations from higher order differential equations", Proceedings of the 42nd IEEE Conference on Decision and Control, Hawaii, pages 1633-1638, 2003.

L. Pernebo and L. M. Silverman, "Model reduction via balanced state space representations," IEEE Trans. Automat. Contr., vol. 27, pp. 382 - 387, April 1982.

Siep Weiland, Theory of Approximation and Disturbance Attenuation for Linear System, Ph.D. Thesis, Rijksuniversiteit Groningen, 1991.

J. C. Willems, P. Rapisarda, "Balanced state representations with polynomial algebra", Springer Lecture Notes in Control and Information Sciences, 286, pages 345$357,2002$.

Willems, J.C. and Trentelman, H.L., "The dissipation inequality and the algebraic Riccati equation", The Riccati Equation, Springer Verlag, 1991.

Willems, J.C. and Trentelman, H.L., "On quadratic differential forms", SIAM J. Control Opt., vol. 36, no. 5, pp. 1703-1749, 1998.

J.C. Willems and H.L. Trentelman, "Synthesis of dissipative systems using quadratic differential forms - part I", IEEE Transactions on Automatic Control, vol. 47, nr. 1, pp. 53 - 69, 2002.

\section{CONCLUSIONS}

We close this paper with some conclusions: 